Obere Extremität 2014 · 9:1

DOI 10.1007/s11678-014-0249-1

Online publiziert: 21. Februar 2014

c) Springer-Verlag Berlin Heidelberg 2014

Thilo Patzer

Orthopädische Klinik, Universitätsklinikum Düsseldorf, Düsseldorf, Deutschland

\title{
Läsionen der langen Bizepssehne, SLAP- und Bizeps-pulley-Läsionen
}

Mobilität des Labrums im Bereich des anterosuperioren Quadranten mit gehäuftem Vorliegen eines sublabralen Rezessus oder Foramens zu berücksichtigen. Fixationen der Normvarianten resultieren oft in Schultersteifen und limitierter Schulterfunktion. Augenmerk sollte auf die Fixation der LBS-Insertion und des posterosuperioren Labrums gelegt werden. Ob hier ein bzw. zwei Fadenanker oder knotenlose Anker verwendet werden sollen, ist diskutabel, vertikale Nähte scheinen hierbei zu dominieren. Die Erfolge des pulley-Repairs sind jedoch eher limitiert und münden häufig in einer statischen LBS-Fixation im Sinne einer Tenodese.

Die LBS-Tenodese stellt eine gute Therapieoption für LBS-Läsionen dar. Sie ermöglicht es sogar Leistungssportlern, auf das ursprüngliche sportliche Niveau zurückzukehren. Wenn man sich für die Tenodese entscheidet, sollte man eine suffiziente Technik verwenden. Wichtig ist hierbei, ein Impingement der Sehne durch epiossäres Aufliegen der Sehne oder Knoten zu vermeiden und ein intraossäres Einwachsen der Sehne zu ermöglichen. Als Goldstandard wird die intraossäre Sehnenfixation mit einer Interferenzschraube angesehen. Diese Technik ist subpektoral und suprapektoral, offen oder arthroskopisch möglich, wobei das glenohumerale von dem subakromialen arthroskopischen Vorgehen unterschieden wird. Nach wie vor wurde außer der erhöhten Inzidenz von „Popeye-sign“-Deformitäten des Oberarms nach LBS-Tenotomie kein funktioneller Vorteil der LBSTenodese im Vergleich zur -Tenotomie nachgewiesen. Allerdings mangelt es an prospektiv randomisierten Vergleichen der Tenotomie mit suffizienten Tenode- setechniken. Aufgrund kosmetischer Defizite im Bereich des Oberarms und aufgrund prolongierter Muskelkrämpfe des Bizeps nach Tenotomie empfiehlt sich bei jüngeren Patienten, eine Tenodese durchzuführen.

Zusammenfassend können LBS-Läsionen häufig sowohl glenohumerale Schmerzen als auch typisch lokalisierte humerale Chondralläsionen verursachen. Als langfristig sichere therapeutische Option hat sich die LBS-Tenodese/ -Tenotomie herausgestellt, um den „Störfaktor" der instabilen LBS aus dem Gelenk zu beseitigen und Sekundärschäden zu verhindern.

Es besteht jedoch ein Bedarf an prospektiv randomisierten Studien zur Evaluation einer eindeutigen Evidenz für die Vorteile der LBS-Tenodese gegenüber der -Tenotomie und für die mögliche Definition der LBS-Läsionen als früharthrotischer Pathogenitäts-Faktor für das Glenohumeralgelenk, um uns ein abschließendes Urteil zu erlauben.

Ihr

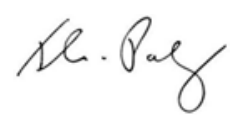

Thilo Patzer

\section{Korrespondenzadresse}

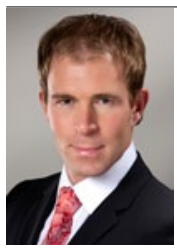

Dr. T. Patzer

Orthopädische Klinik Universitätsklinikum Düsseldorf, Moorenstraße 1-5 40225 Düsseldorf thilo.patzer@med.uni-duesseldorf.de werden sollten. Konsens besteht darin, bei der SLAP-Refixation die physiologische 